\title{
BMJ Open Contextual factors associated with life- space mobility in community-dwelling older adults based on International Classification of Functioning, Disability and Health: protocol for a systematic review
}

\author{
Heloisa Maria Jácome Sousa Britto, ${ }^{1}$ Bruna Silva Oliveira, ${ }^{1}$ \\ Cristiano Santos Gomes, ${ }^{1}$ Juliana Martins Pinto, ${ }^{2}$ Ricardo Oliveira Guerra ${ }^{1}$
}

To cite: Britto HMJS, Oliveira BS, Gomes CS, et al. Contextual factors associated with life-space mobility in communitydwelling older adults based on International Classification of Functioning, Disability and Health: protocol for a systematic review. BMJ Open 2018;8:e023468. doi:10.1136/ bmjopen-2018-023468

- Prepublication history and additional material for this paper are available online. To view these files, please visit the journal online (http://dx.doi. org/10.1136/bmjopen-2018023468).

Received 8 April 2018

Revised 9 July 2018

Accepted 18 September 2018

Check for updates

(c) Author(s) (or their employer(s)) 2018. Re-use permitted under CC BY-NC. No commercial re-use. See rights and permissions. Published by BMJ.

1Department of Physiotherapy, Federal University of Rio Grande do Norte, Natal, Rio Grande do Norte, Brazil

${ }^{2}$ Department of Physiotherapy, Federal University of Triangulo Mineiro, Uberaba, Minas Gerais, Brazil

Correspondence to Dr Heloisa Maria Jácome Sousa Britto;

fsthelobritto@gmail.com

\section{ABSTRACT}

Introduction Mobility decline compromises functionality and quality of life in old age. Life-Space Assessment (LSA) evaluates mobility considering interaction between person and environment. The International Classification of Functioning, Disability and Health (ICF) is a reference to identify and categorise the personal and environmental contextual factors associated to the LSA. Our objective is identifying contextual factors that may influence life-space mobility of older community-dwelling adults based on ICF. Methods and analysis A systematic review of literature will be performed to identify studies published between 1 January 2001 and 10 May 2017 which investigates life-space mobility among older adults. Keywords will be entered into the electronic databases of MEDLINE (PubMed), EMBASE (OVID), CINHAL (EBSCO), Cochrane Central Register of Controlled Trials Cochrane Central (OVID), PsycINFO (EBSCO) and COCH (OVID). Five investigators will work on search databases and standardised screening of the articles. Mobility predictors will be separated into personal and environmental aspects, according to the ICF model. The results will be reported according to the Preferred Reporting Items for Systematic Reviews and Meta-Analyses statement, and then a metaanalysis will be performed, if applicable.

Discussion Knowledge about life-space mobility in community-dwelling older adults by examining related risk and protective aspects may help practitioners better approach older adults' mobility and prevent their decline in old age. Furthermore, researchers will have more clues for investigations into factors related to life-space mobility. Trial registration number CRD42017064552.

\section{INTRODUCTION}

Mobility has been defined as the ability to move oneself (either independently or by using assistive devices or transportation) within environments that expand from one's home to the neighbourhood and to regions beyond. It is possible to understand mobility

\section{Strengths and limitations of this study}

- The article proposes to analyse the life-space mobility in community-dwelling older adults based on International Classification of Functioning, Disability and Health.

- Full-text screening will be independently conducted by two reviewers using a data collection standardised form.

- The contextual factors that may influence the decline in mobility among older adults can be a key point for possible intervention strategies.

- The study only described a protocol ongoing study.

in a holistic way. ${ }^{1}$ According to Webber et $a l,{ }^{1}$ in 'Mobility in Older Adults: A Comprehensive Framework', the mobility key determinants include cognitive, psychosocial, physical environmental, and financial influences.

The Life-Space Assessment (LSA) is a reliable and validated instrument to assess self-reported mobility which evaluates life-space mobility from an ecology perspective ${ }^{2}$; it is a promising component to guide intervention programmes and a popular item among scientists and gerontologists who conduct research on ageing linked to quality of life and healthy and active ageing. ${ }^{3-5}$

The LSA has been widely associated with different areas and health conditions in older people such as hospitalisation, ${ }^{6}$ cognitive function, ${ }^{78}$ falls, ${ }^{9}$ muscle atrophy, ${ }^{10}$ faecal incontinence, ${ }^{11}$ oral health-related quality of life ${ }^{12}$ and frailty. ${ }^{13}$ Other studies have reported associations between LSA and contextual aspects such as characteristics of the physical environment and social networks. ${ }^{21415}$ 


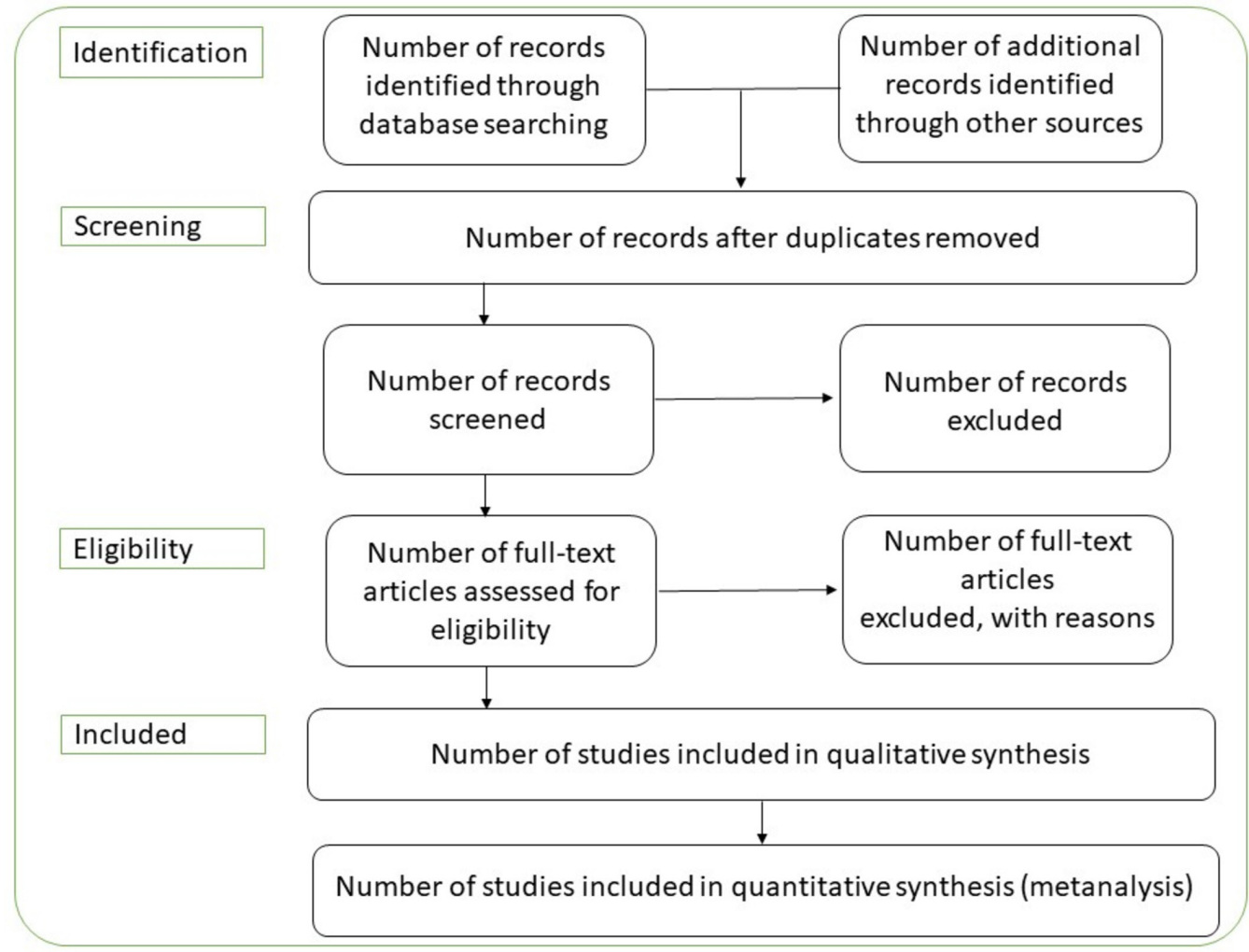

Figure 1 Preferred Reporting Items for Systematic Reviews and Meta-Analyses (PRISMA) flow diagram.

Auais $e t a l^{16}$ evaluated 1995 community-dwelling older adults in a multicentric study and identified the average LSA total score as 68.7 (SD: 21.2), while its distribution was significantly different across the five study sites $(\mathrm{p}<0.001)$ with averages ranging from 56.6 in Natal, Brazil to 82.6 in Kingston, Canada. This indicates that more developed cities presented better scores.

Although these associations have been studied, there has not yet been a systematic analysis of LSA on the International Classification of Functioning, Disability and Health (ICF) perspective.

The ICF was developed by the WHO in $2001,{ }^{17}$ and describes human functioning and disability as a dynamic interaction between health status (diseases, disorders, injuries, traumas, etc), body structure and function, activity and participation within contextual factors. According to the ICF, the contextual factors include the complete history of individuals' lives and lifestyles, comprising personal and environmental factors. ${ }^{17}$

Considering that the ICF is a valuable reference and framework for health outcome measurement, ${ }^{17}$ we will use the ICF to identify and categorise the personal and environmental factors associated to LSA. This approach could help increase the clinical relevance and to internationally provide stronger evidence of the impact of LSA in older people.

\section{OBJECTIVES}

Our objective is to explore studies about LSA in community-dwelling older adults by performing a systematic review to identify the variables associated to LSA, then to classify them according to ICF structure in order to understand which contextual factors may influence the life-space mobility of community-dwelling older adults. Our hypothesis is that both environmental and personal aspects impact older adults' mobility.

\section{METHODS AND ANALYSIS}

The current protocol has been registered in the PROSPERO database (registration number: CRD42017064552). The systematic review will follow the four-phase flow diagram (figure 1) put forth by the Preferred Reporting Items for Systematic Reviews and Meta-Analyses (PRISMA) statement. ${ }^{18}$

\section{SEARCH STRATEGIES}

Literature searches will be performed by the research team (HMJSB, BSO, CSG, JMP and ROG) from the Federal University of Rio Grande do Norte. We will use specific medical subject headings $(\mathrm{MeSH})$ and words from 'all fields' to identify studies in MEDLINE (PubMed interface), EMBASE (OVID interface), CINHAL (EBSCO interface), Cochrane Central Register of Controlled Trials Cochrane Central (CCTR) (OVID interface), PsycINFO (EBSCO interface) and COCH (OVID interface) from their start date onwards. Details concerning search strategy are provided in online supplementary file 1 . We will also search through a grey literature source (Google Scholar). Furthermore, we will search official websites of 
active institutions in the fields of life-space mobility and/ or life-space/ageing, along with bibliographic references of retrieved articles and relevant reviews.

The search strategy will combine the following themes or theme groups: (1) Elderly/Ageing; (2) Mobility; (3) 'Life-space Assessment'; (4) Questionnaire; (5) Environment. All articles and reports retrieved during the screening phase will be combined in an Endnote file, and duplicates will be extracted.

The complete search strategies are found in online supplementary file 1 .

\section{Study inclusion criteria: participants and setting}

Each study should meet the following criteria:

1. Sample composed of community-dwelling older adults aged 65 years or older.

2. Observational studies (cohort, case-control, cross sectional) and randomised controlled trial (RCT).

3. Mobility evaluated through life-space measurement (measurement criterion).

4. Papers published in Portuguese, English and Spanish (language criterion).

5. Papers published between 1 January 2000 and 10 May 2017.

6 . The objective of the research is to determine the association between life-space mobility and contextual aspects (environmental and personal) or evaluated predictors, factors and determinants of life-space mobility (relationship criterion).

\section{Primary outcomes}

LSA association with the following environmental contextual aspects (physical characteristics of the environment; interpersonal relations; legislation; use of products and technology) and personal contextual aspects (economic conditions; life course adversities; employability; attendance to medical appointments or hospitalisation; gender; race; age; lifestyle; different ways of facing problems; social background; level of education).

\section{Secondary outcomes}

LSA association with aspects related to body structure and function, activity and participation: sensory, physical or mental illness, deficiencies or deficits, physical capacity, physical performance, activities of daily living and move around using transport.

\section{ANALYSIS STRATEGIES \\ Study selection procedure}

We will perform a standard data extraction template to extract the main details for every eligible study in terms of author, year, title, objective, sample size, country, design, life-space mobility measurement and main results.

The search process will be carried out by two researchers (CSG and HMJSB) and an expert librarian. Two researchers (HMJSB and BSO) will examine each title and abstract to identify a potentially relevant study for review. Articles will be classified as: (1) included, (2) excluded or (3) uncertain using the 'covidence' site. The full text of articles from selected abstracts (records categorised as included or uncertain) will be obtained for further eligibility analysis. In case of uncertainty, a third researcher (JMP) will check the selection process. Full-text screening will be independently conducted by two reviewers (HMJSB and BSO) using a data collection standardised form with explicit inclusion and exclusion criteria (see online supplementary file 2). Discrepancies in eligibility will be resolved by two reviewers. In the event of an unsettled disagreement, the opinion of a senior epidemiologist will be required (ROG).

\section{Data collection process}

Reviewers will fill out an explicit data collection form to organise study characteristics (country, setting, year of publication, study design and sample size); participants' characteristics (age, gender, body mass index, socioeconomic position and health status); and outcomes (factors which can be related to LSA in community-dwelling older adults). Reviewers will systematically use a standardised data abstraction form (see online supplementary file 2). HMJSB and BSO will independently extract the data. Unsettled disagreements will be resolved by a senior reviewer (ROG and JMP).

\section{Scientific quality assessment}

Scientific quality of selected articles will be appraised using a standardised quality assessment tool tailored to best appraise the studies' methodological quality and bias, the Cochrane Collaboration's Risk of Bias Tool will be used for RCTs and the Strengthening the Reporting of Observational Studies in Epidemiology will be used for observational studies.

\section{Data synthesis}

If meta-analyses are not possible, data will be narratively synthesised. As reported above, the results will be reported according to the PRISMA statement. ${ }^{18}$ Evidence tables will be generated to descriptively summarise the included studies and results: (1) authors, (2) study design, (3) age group, (4) country, (5) objectives, (6) population, (7) recruitment, (8) life-space mobility score, (9) environmental and personal contextual aspects, (10) aspects related to body structure and function, and (11) scientific quality.

Finally, the ICF domains will be grouped and analysed by primary outcomes (environmental and personal contextual factors) and secondary outcomes (health conditions, body structure and function, activity and participation).

\section{Quantitative synthesis}

Results from each study will be compiled in summary tables for descriptive comparisons of study findings. As primary outcomes we will evaluate LSA association with environmental and personal contextual aspects. We will aggregate study findings for each exposure in order to perform meta-analyses assessing overall magnitude of 
the association with life-space mobility measurement. We recognise that there are variations for measuring each of our exposures, which will require us to stratify our findings to better account for study heterogeneity.

\section{Ethics and dissemination}

No ethical issues are foreseen. The findings of this review will be submitted for publication in a peer-reviewed journal, and this paper is included in the lead author's doctoral dissertation.

\section{Patient and public involvement}

Patients and or public were not involved.

\section{DISCUSSION}

This systematic review will provide:

1. Knowledge about the factors associated with mobility in the life-space in order to identify the environmental aspect influences on the decline of mobility in old age.

2. A scientific basis sufficient to understand the influences for the decline of life-space mobility in ageing. Identifying contextual aspects associated with mobility deficit may help clinical research and preventive medical conduct.

3. Generating knowledge on environmental and personal contextual aspects can guide the government to insert public policies directed towards active ageing.

4. Disseminate the use of the LSA as a measurement tool for life-space mobility of older adults, including in primary care centres.

The contextual factors that may influence the decline in mobility among older adults can be a key point for possible intervention strategies, guidelines based on health education, or even policymaking to minimise the decline in mobility for older adults in the community. It is also hoped that the knowledge from this review will be used for exemplifying contextual aspects of the ICF and its impact on older populations, as well as for direct future clinical research in testing changes on contextual factors as a modifiable variable of health in life-space mobility.

We believe that knowledge about environmental and personal contextual aspects can guide the government to insert public policies directed at active ageing, seeing that from the findings of this study we will be able to identify which variables influence mobility decline in older adults. Thus, this could be used by public health managers to formulate strategies to minimise damage of contextual factors which affect mobility and thereby provide better life-space mobility in older adult communities.

Finally, this systematic review could contribute to disseminate the use of the LSA as a measurement tool of life-space mobility for older adults, including in primary care centres. The LSA is a tool that is associated with important aspects of senility such as falls, ${ }^{19}$ frailty,${ }^{20}$ cognitive decline, ${ }^{8}$ and is a mortality predictor, ${ }^{21}$ as well as being a cheap and easy-to-use instrument, so it is possible that a primary care centre in health can use this tool to evaluate older adults. LSA use may be used to alert professionals because it can identify an older adult with a low score, and thereby alert health professionals to an individual(s) who needs more attention and care in order to minimise the problems of senility.

Contributors HMJSB and BSO conceived and designed the protocol and drafted the manuscript. HMJSB, BSO, JMP and ROG participated in the critical revision of the manuscript for methodological and intellectual content. HMJSB and CSG carried out the search process. All authors approved the final version of the manuscript.

Funding The authors have not declared a specific grant for this research from any funding agency in the public, commercial or not-for-profit sectors.

Competing interests Noen declared.

Patient consent Not required.

Provenance and peer review Not commissioned; externally peer reviewed.

Open access This is an open access article distributed in accordance with the Creative Commons Attribution Non Commercial (CC BY-NC 4.0) license, which permits others to distribute, remix, adapt, build upon this work non-commercially, and license their derivative works on different terms, provided the original work is properly cited, appropriate credit is given, any changes made indicated, and the use is non-commercial. See: http://creativecommons.org/licenses/by-nc/4.0/.

\section{REFERENCES}

1. Webber SC, Porter MM, Menec VH. Mobility in older adults: a comprehensive framework. Gerontologist 2010;50:443-50.

2. Peel C, Sawyer Baker P, Roth DL, et al. Assessing mobility in older adults: the UAB Study of Aging Life-Space Assessment. Phys Ther 2005;85:1008-119.

3. Lo AX, Flood KL, Kennedy RE, et al. The Association between lifespace and health care utilization in older adults with heart failure. $J$ Gerontol A Biol Sci Med Sci 2015;70:1-6.

4. Rantakokko M, Portegijs E, Viljanen A, et al. Changes in life-space mobility and quality of life among community-dwelling older people: a 2-year follow-up study. Qual Life Res 2016;25:1189-97.

5. Portegijs E, Tsai L-T, Rantanen T, et al. Moving through life-space areas and objectively measured physical activity of older people. PLoS One 2015;10:e0135308.

6. Brown CJ, Roth DL, Allman RM, et al. Trajectories of life-space mobility after hospitalization. Ann Intern Med 2009;150:372-8.

7. O'Connor ML, Edwards JD, Wadley VG, et al. Changes in mobility among older adults with psychometrically defined mild cognitive impairment. J Gerontol B Psychol Sci Soc Sci 2010;65B:306-16.

8. Crowe M, Andel R, Wadley VG, et al. Life-space and cognitive decline in a community-based sample of African American and Caucasian older adults. J Gerontol A Biol Sci Med Sci 2008;63:1241-5.

9. Lo AX, Rundle AG, Buys D, et al. Neighborhood Disadvantage and Life-Space Mobility Are Associated with Incident Falls in CommunityDwelling Older Adults. J Am Geriatr Soc 2016;64:2218-25.

10. Ikezoe T, Mori N, Nakamura M, et al. Age-related muscle atrophy in the lower extremities and daily physical activity in elderly women. Arch Gerontol Geriatr 2011;53:e153-7.

11. Goode PS, Burgio KL, Halli AD, et al. Prevalence and correlates of fecal incontinence in community-dwelling older adults. J Am Geriatr Soc 2005;53:629-35.

12. Makhija SK, Gilbert GH, Clay OJ, et al. Oral health-related quality of life and life-space mobility in community-dwelling older adults. J Am Geriatr Soc 2011;59:512-8.

13. Xue QL, Fried LP, Glass TA, et al. Life-space constriction, development of frailty, and the competing risk of mortality: the Women's Health And Aging Study I. Am J Epidemiol 2008;167:240-8.

14. Al Snih S, Peek KM, Sawyer P, et al. Life-space mobility in Mexican Americans aged 75 and older. J Am Geriatr Soc 2012;60:532-7.

15. Shimada $H$, Ishizaki $T$, Kato $M$, et al. How often and how far do frail elderly people need to go outdoors to maintain functional capacity? Arch Gerontol Geriatr 2010;50:140-6.

16. Auais $M$, Alvarado $B$, Guerra $R$, et al. Fear of falling and its association with life-space mobility of older adults: a cross-sectional analysis using data from five international sites. Age Ageing 2017;46:459-465.

17. WHO. The International classification of functioning, disability and health. World Heal Organ 2001;18:237. 
18. Liberati A, Altman DG, Tetzlaff J, et al. The PRISMA statement for reporting systematic reviews and meta-analyses of studies that evaluate healthcare interventions: explanation and elaboration. BMJ 2009;339:b2700.

19. Lo AX, Brown CJ, Sawyer P, et al. Life-space mobility declines associated with incident falls and fractures. J Am Geriatr Soc 2014;62:919-23.
20. Portegijs E, Rantakokko M, Viljanen A, et al. Is frailty associated with life-space mobility and perceived autonomy in participation outdoors? A longitudinal study. Age Ageing 2016;45:550-3.

21. Kennedy RE, Sawyer P, Williams CP, et al. Life-Space mobility change predicts 6-month mortality. J Am Geriatr Soc 2017;65:833-8. 